\title{
Metanálise da relação entre a performance social e a performance financeira corporativa
}

\author{
Meta-analysis of the relation between corporate social \\ performance and corporate financial performance
}

\author{
Ralph Santos da Silva ${ }^{1}$ \\ João Maurício Gama Boaventura² \\ Rodrigo Bandeira-de-Mello ${ }^{3}$
}

\begin{abstract}
Resumo: Segundo a teoria dos stakeholders, empresas que coordenam os interesses dos diversos stakeholders apresentam um desempenho superior àquelas que priorizam apenas os interesses dos shareholders e, portanto, um melhor desempenho social corporativo - CSP, entenda-se melhor atendimento dos interesses dos stakeholders, leva a um melhor desempenho financeiro corporativo - CFP. O interesse desse tema desencadeou a realização de dezenas de pesquisas empíricas testando a relação entre CSP e CFP. No início da década de 2000, a predominância dos estudos empíricos revelava uma associação positiva entre CSP e CFP e, em função disso, a comunidade acadêmica aceitou ser essa relação normalmente positiva. Entretanto, ao menos duas questões chamam atenção: "A predominância da relação positiva entre CSPe CFP ainda persiste em pesquisas mais recentes?" e "Quais stakeholders estão mais associados positivamente ao desempenho financeiro?" Em função dessas questões, o objetivo desta pesquisa é a análise da relação CSP-CFP a partir das pesquisas empíricas dos últimos anos. Empregou-se a técnica de metanálise aplicando-a aos dados dos artigos empíricos publicados entre 1998 e 2010. Os resultados não confirmam uma generalização da relação positiva entre a CSP e a CFP, além disso, na análise individual, as relações entre o desempenho junto a cada stakeholder e o desempenho financeiro, apenas o stakeholder funcionário apresentou clara relação positiva. Esses resultados sugerem mais cautela no uso gerencial da premissa de uma relação CSP-CFP positiva e a recomendação de mais estudos quanto às nuances dessa relação.
\end{abstract}

Palavras-chave: Stakeholder; Responsabilidade social; Performance social; Performance financeira; Metanálise.

\begin{abstract}
According to the stakeholder theory, companies which coordinate the interests of their several stakeholders show better performance than companies which prioritize only shareholders' interests, and thus also show a better corporate social performance (CSP, or better addressing stakeholders' interests), which leads to a better corporate financial performance (CFP). The interest in this subject has elicited a series of empirical studies testing the relation between CSP and CFP. In the beginning of the 2000's most part of those studies revealed a positive association between CSP and CFP and consequently academia has taken it to be generally positive. However at least two issues deserve closer examination: "Does the prevalence of a positive relation between CSP and CFP still hold in more recent research?" and "Which stakeholders are more positively associated to the financial performance?" The aim of the present paper is to analyze the relation between CSP and CFP in the light of empirical research in the last few years. The meta-analysis technique has been employed on articles published between 1998 and 2010. Results do not confirm generalization of a positive relation between CSP and CFP. Furthermore, when the link between each stakeholder's performance and respective financial performance was tested, only the "employee" stakeholder presented a clear positive relation. Such results suggest a more cautious use of the positive CSP/CFP relation by company management, as well as the pursuit of further studies on the nuances of this relation.
\end{abstract}

Keywords: Stakeholder; Social responsibility; Social performance; Financial performance; Meta-analysis.

\section{Introdução}

No nível estratégico, Slack et al. (2002) classificam os objetivos de desempenho da produção em amplos e específicos. Os objetivos amplos são relacionados aos interesses dos stakeholders (colaboradores, fornecedores, clientes, acionistas, entre outros) e os específicos são relacionados diretamente às

\footnotetext{
${ }^{1}$ Centro de Tecnologia da Informação - CTI, Rodovia Dom Pedro I, Km 143,6, Techno Park, CEP 13092-599, Campinas, SP, Brasil, e-mail: silva.ralph@gmail.com

${ }^{2}$ Programa de Pós-graduação em Administração - PPGA, Faculdade de Economia, Administração e Contabilidade - FEA, Universidade de São Paulo - USP, Av. Prof. Luciano Gualberto, 908, Butantã, CEP 05508-010, São Paulo, SP, Brasil, e-mail: jboaventura@usp.br

${ }^{3}$ Departamento de Administração Geral e Recursos Humanos, Escola de Administração de Empresas de São Paulo - EAESP, Fundação Getúlio Vargas - FGV, Av. Nove de Julho, 2029, Bela Vista, CEP 01313-902, São Paulo, SP, Brasil, e-mail: rodrigo.mello@fgv.br
} Recebido em Out. 9, 2016 - Aceito em Jun. 3, 2017

Suporte financeiro: Conselho Nacional de Desenvolvimento Científico e Tecnológico - CNPq. 
decisões estruturais e de infraestrutura, conforme definem Hayes \& Wheelwright (1984). Apesar de explicitarem a necessidade de atender aos interesses dos stakeholders, Slack et al. (2002) não explicitam nenhuma relação de precedência entre $o$ atendimento de interesses de diferentes grupos de stakeholders. Portanto, não fica claro se atender aos interesses dos acionistas tem ou não precedência sobre atender aos interesses dos colaboradores, cliente e fornecedores, ou vice-versa.

Freeman (1984) é contundente quanto a essa relação de precedência. Sua proposição é de que empresas que atendem aos interesses dos stakeholders apresentam desempenho financeiro superior em relação àquelas que buscam a atender apenas aos interesses dos acionistas. Essa proposição suscitou e ainda suscita uma intensa busca de evidências empíricas para sua sustentação. Uma decorrência desse argumento é que uma melhor performance social corporativa - CSP, ou seja, melhor atendimento dos interesses dos stakeholders, teria melhor performance financeira corporativa - CFP.

O impacto para a gestão das empresas ao adotarem a visão da teoria dos stakeholders baseada na premissa de uma relação positiva entre CSP e CFP levou dezenas de cientistas nas décadas de 80 e 90 ao desenvolvimento de dezenas de pesquisas empíricas testando a relação entre CSP e CFP. Ao início da década de 2000, a academia discutia intensamente qual contribuição teórica sobre essa relação seria possível com base nas pesquisas empíricas. São então publicados dois trabalhos, os quais se tornaram referência para esse campo de pesquisa, que consolidaram, por meio de metanálises, os achados dos estudos empíricos: Margolis \& Walsh (2003) e Orlitzky et al. (2003). Cabe ressaltar que o primeiro deles consistiu em uma metanálise qualitativa e o segundo, estatística. Tais trabalhos levaram a um reforço do entendimento de que a relação CSP-CFP é positiva, assim como a respectiva proposição da teoria dos stakeholders.

Entretanto, seria razoável admitir que, passados mais de 10 anos dos trabalhos de Margolis \& Walsh (2003) e Orlitzky et al. (2003), os resultados dos estudos empíricos dessa relação CSP-CFP se mantêm? O presente estudo se justifica, primeiramente, por atualizar essa análise, buscando estudar se ainda permanecem tais resultados e por responder objetivamente as questões: "A predominância da relação positiva entre CSP e CFP ainda persiste em pesquisas mais recentes?" e "Quais stakeholders estão mais associados positivamente à performance financeira?" Reforçam a justificativa desta pesquisa estudos mais recentes, a exemplo de Van Beurden \& Gössling (2008), que pontuam exatamente a necessidade de se estudar melhor essa relação em função das várias nuances existentes nessa relação.
Com base nessas questões de pesquisa definiu-se o objetivo deste estudo: analisar a relação entre a Corporate Social Performance (CSP) e a Corporate Financial Performance (CFP), considerando os trabalhos empíricos já publicados nos últimos anos. Para operacionalizar essa pesquisa se recorreu a técnica estatística de metanálise. A metanálise, conforme propõem Hunter \& Schmidt (1990), se baseia em dados empíricos de artigos publicados no período de tempo de interesse. Com base em uma série acumulada de trabalhos, a metanálise permite estabelecer conclusões de nível mais geral e de forma agregada. Dessa maneira também é possível tratar os dados conjuntamente, no sentido de retirar possíveis distorções postas pelos próprios instrumentos de pesquisa dos trabalhos originais.

Este artigo enfoca a relação CSP-CFP e pretende contribuir no sentido de apurar a natureza dessa relação não só analisando-a em nível de proxies, mas também explorando-a em termos de indicadores que as representam.

Independentemente da importância da discussão teórica da relação entre CSP-CFP e da análise dos diversos estudos empíricos pertinentes, este estudo também tem o propósito de contribuir metodologicamente. Cabe pontuar que poucos são os estudos de metanálise estatística realizados na relação CSP-CFP. Nesse particular, o presente estudo visa apresentar esse método de uma forma didática e replicável, evidenciando os passos necessários para o emprego dessa técnica em futuros estudos neste campo de pesquisa.

\section{Referencial teórico}

O tema deste estudo aborda a relação entre performance social corporativa-CSP e performance financeira corporativa - CFP, construtos que se entrelaçam com conceitos de responsabilidade social corporativa - CSR, da teoria dos stakeholders e da teoria da firma. A explanação a seguir visa apresentar as relações entre os conceitos de CSR, CSP e CFP no âmbito das duas teorias citadas. Em especial, a explanação do entrelaçamento entre CSP e CSR aqui se justifica, pois para fins deste estudo ambas podem ser associadas com a função objetivo da teoria dos stakeholders de coordenar o interesse dos stakeholders. Observe-se que em diversos artigos empíricos analisados essa associação não se fez explícita, uma vez que o objetivo dos mesmos não eram necessariamente os mesmos deste estudo.

\subsection{A Responsabilidade social corporativa - CSR}

Como apresentado em Whetten et al. (2002), a responsabilidade social corporativa a partir dos anos 50 se desenvolveu por meio de ações ingênuas 
implementadas pelas organizações, até que se estabeleceu de forma estruturada pela contratação de profissionais para lidar com problemas específicos de meio ambiente, comunidades, clientes, empregados. Em 1971, Mazis \& Green (1971), num artigo focando a responsabilidade social corporativa, sugerem a criação de comitês para serem utilizados na sua gestão, contando com a participação de gerentes e representantes de departamentos ligados às questões sociais e tornando evidente, dessa forma, que muitas organizações estariam efetivamente gerenciando aspectos específicos inclusos no contexto da responsabilidade social corporativa.

Carroll (1979) define a CSR (Corporate Social Responsability) como sendo o espectro de obrigações que o negócio tem para com a sociedade, englobando quatro categorias: o econômico, o legal, o ético e o discricionário. Essas quatro categorias devem ser vistas de maneira simultânea, implicando que a empresa deve ter responsabilidade e ações sobre as categorias. A responsabilidade econômica aponta para a responsabilidade básica que é a de produzir produtos e serviços que atendam as necessidades da sociedade, e a sua venda deve gerar lucros para a empresa. A responsabilidade legal aponta para os aspectos contratuais entre as partes, que devem ser respeitadas. A responsabilidade ética incorpora normas e comportamentos que não são necessariamente cobertos pelos aspectos legais, mas que igualmente devem ser atendidos pelas partes. E a responsabilidade discricionária aponta para as ações voluntárias promovidas pelos gerentes e que nem sempre são entendidas pela sociedade.

Estudos empíricos que buscam avaliar a performance de CSR normalmente o fazem definindo CSR como uma proxy dependente de variáveis que se relacionam com um amplo espectro de stakeholders, como empregados, clientes, investidores, fornecedores, comunidade e meio-ambiente. Exemplos disso podem ser observados nos trabalhos de Mishra \& Suar (2010), Chih et al. (2010), Fauzi \& Idris (2009) e Lougee \& Wallace (2008). Em função disso é possível associar a variável CSR desses estudos empíricos com o atendimento da função objetivo da teoria dos stakeholders.

\subsection{Performance social corporativa - CSP}

Além de definir a CSR, Carroll (1979) articula o processo de responsividade social (social responsiveness) e a dimensão resultados (outcomes) no sentido de incorporá-los em um modelo da CSP (Corporate Social Performance). Dessa forma, buscando atender demandas conceituais que suportam a prática da responsabilidade social. Em seu modelo está proposto que o efetivo desempenho nessa arena requer dos gerentes: a identificação sobre os temas, os quais estão
Quadro 1. O modelo de performance social corporativa.

Princípios da responsabilidade social corporativa

Princípio institucional: Legitimidade

Princípios organizacionais: Responsabilidade pública

Princípio individual: Discrição gerencial

Processos da responsividade social corporativa

Avaliação ambiental

Gestão de stakeholder

Gerenciamento de tópicos

Resultados do comportamento corporativo

Impacto social

Programas sociais

Políticas sociais

Fonte: Wood (1991).

postos para a organização; a reflexão que esses tipos de responsabilidades sociais convocam; a seleção do modo pela qual a responsividade será estabelecida (reativa, defensiva, acomodativa, proativa); e o tipo de resultado esperado. Sua proposta suporta diversos trabalhos posteriores, como será tratado a seguir.

Wood $(1991,2010)$ partindo da proposta de Carroll (1979), estabelece um modelo ampliado pela incorporação de contribuições de outros trabalhos que também se propuseram a delinear a CSP. Sua proposta pode ser representada conforme o Quadro 1.

Wood (1991) argumenta que para avaliar a CSP, a pesquisa poderia examinar em que grau os princípios da responsabilidade social motivam ações de interesse da empresa, em que grau as empresas fazem uso de processos responsivos e qual a natureza das políticas e programas desenhados pela gestão em termos de relacionamentos societais e dos impactos (observáveis) gerados por essas ações, programas e políticas.

Ao se analisar como os estudos empíricos modelam a variável CSP observa-se que essa normalmente é definida como uma proxy que se associa a variáveis referentes a diversos stakeholders. Isso pode ser constatado, por exemplo, nos trabalhos de Lee et al. (2009), Callan \& Thomas (2009) e Mahoney et al. (2008). Nesse sentido, assim como nos estudos empíricos que modelam uma variável para CSR, essa variável CSP também é um medidor de coordenação dos interesses dos stakeholders e da função objetivo da teoria dos stakeholders.

\subsection{Teoria dos stakeholders}

A teoria dos stakeholders (Freeman, 1984) emerge nos anos 80 não como uma teoria mas como um conceito usual para comunicar a necessidade de gerenciar os relacionamentos com pessoas ou grupos de pessoas para organizações preocupadas com temas sociais, não necessariamente com temas econômicos identificados pelo campo da estratégia tal qual Porter (1980). Nos anos 90, entretanto, o conceito de stakeholder move-se em direção a uma teoria mais completa e torna-se um importante referencial teórico nesse campo de pesquisa (Whetten et al., 2002). 
Segundo Frooman (1999), a principal referência dessa teoria encontra-se na obra de Freeman de 1984. Coombs \& Gilley (2005) esclarecem que a definição do termo stakeholder mais empregada na literatura é também a de Freeman (1984) e estabelece que stakeholder é qualquer indivíduo ou grupo que possa afetar a obtenção dos objetivos organizacionais ou que é afetado pelo processo de busca desses objetivos. Pela óptica da teoria dos stakeholders, o objetivo da empresa, segundo Evan \& Freeman (1993, p. 170), é "[...] servir de veículo para coordenar os interesses dos stakeholders".

O gerenciamento dos relacionamentos com os stakeholders é visto como sendo de significado mais robusto, conceitualmente, para os estudos das ações organizacionais no domínio social do que a abordagem dos temas sociais (Clarkson, 1995). Whetten et al. (2002) sugerem que a qualidade dos relacionamentos com um conjunto primário (econômico) e secundário (social) de stakeholders pode de fato ser sinônimo da qualidade do gerenciamento. Para isso esses autores apontam trabalhos anteriores como os de Clarkson (1995), Waddock \& Graves (1997) e Wood \& Jones (1995), que sugerem que a teoria de stakeholder provê uma base adequadamente entendida e accessível do CSP. Wood \& Jones (1995) observam que os stakeholders jogam com três regras fundamentais do CSP: eles são a fonte dos pressupostos do CSP, eles são afetados pelas ações das organizações e eles evoluem na medida em que as empresas atendem os pressupostos do CSP.

Em síntese, a caracterização dos stakeholders proposta por Freeman (1984) associa-se aos princípios, aos processos e aos temas que compõem o modelo de CSP proposto por Wood (1991) e reeditado em Wood (2010). Portanto, como proposto em Clarkson (1995), os stakeholders em si configuram um subconjunto de atores que compõem a sociedade mas possuem como atributo de distinção a capacidade de influenciar e serem influenciados pela empresa.

\subsection{Teoria da firma e a performance financeira corporativa - CFP}

O surgimento da Ciência Econômica, no século XVIII, a partir da obra de Adam Smith sobre as origens da riqueza das nações, é o suporte da origem da teoria da firma. No século XX, diversos estudos, em especial o de Coase (1937), a respeito dos custos de transação, enriqueceram e desenvolveram a teoria da firma.

Para o estudo em questão interessa particularmente a definição da função objetivo da teoria da firma e a associação dela com o conceito de performance financeira corporativa-CFP. Nesse sentido, cabe lembrar que, assim como as demais teorias microeconômicas, a teoria da firma estabelece como objetivo a maximização dos lucros. Entretanto, a maximização do lucro requer definição mais específica, se contábil ou econômico, se de curto ou longo prazo, entre outras características. Jensen (2001) esclarece que, para os economistas, o objetivo da firma deve buscar a maximização do valor de longo prazo, resultante da capacidade dessa de geração de caixa ao longo do tempo.

A performance financeira corporativa - CFP relaciona-se a variação de valor de empresa, em que pesem diferentes formas de definir valor de uma empresa. A associação entre CFP e a teoria da firma se dá em função do objetivo da firma, por essa teoria. Uma vez que por essa óptica teórica o objetivo da firma é a maximização de seu valor, CFP é uma forma de se mensurar essa valoração. O valor da empresa, por sua vez, pode associar-se a diferentes parâmetros, como valor patrimonial (ou valor contábil), valor de mercado ou valor a longo prazo (valor presente líquido) e o CFP apresenta variações em suas métricas que se associam a esses conceitos.

Embora o paradigma contemporâneo da teoria da firma apregoe o valor de longo prazo (Jensen, 2001), ainda há discussões a respeito da melhor alternativa de mensuração (Cochran \& Wood, 1984). Orlitzky et al. (2003) procuram explicar as variações nas formas de mensuração de CFP e após um levantamento na literatura verificaram que CFP vem sendo mensurada basicamente de três formas: medidas de mercado, contábeis e surveys. Esses autores explicam ainda que a primeira abordagem (medidas de mercado) reflete o grau de satisfação dos acionistas, a segunda (medidas contábeis) captura uma idéia da eficiência interna da empresa e a última (survey) provê uma estimativa subjetiva de sua performance financeira.

\subsection{As relações entre CSP e CFP}

A literatura aponta três possibilidades de associação entre a performance social e a financeira: positiva, negativa e neutra. A primeira, suportada pela teoria dos stakeholders, a segunda, pela teoria de firma e a terceira, por estudiosos que não entendem haver essa relação.

A relação positiva é apoiada por pesquisadores da teoria dos stakeholders (Bird et al., 2007). É a visão de Barnett (2007), que afirma que já há substancial embasamento teórico para se explicar que o aumento da CSP promove o aumento de CFP, como explanado por Jones (1995), que empresas que desenvolvem relacionamento de confiança mútua e cooperação com stakeholders obtêm uma vantagem competitiva sobre as que não.

Já a relação negativa é vista por acadêmicos da visão econômica neoclássica. Essa linha de entendimento pode ser observada em Aupperle et al. (1985), para os quais qualquer investimento para promover CSP irá colocar a empresa em desvantagem e irá resultar em uma performance financeira pior. Tal conceito é o mesmo já primeiramente postulado por Friedman (1970), de que há poucos benefícios econômicos para um comportamento social responsável, ao mesmo tempo em que há muitos custos associados a esse comportamento.

O terceiro posicionamento é que não haveria relação entre CSP e CFP. Tal proposta, incialmente colocada por Ullmann (1985), argumenta haver tantos fatores ou 
variáveis que intervêm na relação entre CSP e CFP que, mesmo que por hipótese houvesse uma relação, essa não poderia ser detectada em função dos problemas de mensuração existentes nas pesquisas empíricas que buscam estudar a relação entre CSP e CFP.

Muitos trabalhos empíricos continuam sendo desenvolvidos e os resultados apresentados sobre a relação CSP-CFP continuam diversificados, ou seja, existem trabalhos que encontram relação CSP-CFP com correlação negativa, neutra e positiva.

\subsection{Indicadores de medição de CSR, CSP e CFP}

Alguns pesquisadores dedicaram-se ao desenvolvimento de conceitos para a construção de indicadores de CSR, CSP e CFP. Hopkins (1997), considerando o modelo de CSP desenvolvido por Wood (1991), propõe indicadores que atendam as três dimensões desse modelo: princípios, processos de responsabilidade social (responsividade) e resultados (efeitos internos e externos para os stakeholders e efeitos institucionais externos). Clarkson (1995), por sua vez, propõe associar esses indicadores a temas ligados ao gerenciamento do stakeholder. Esses temas estão sob seis grandes tópicos: a empresa, os empregados, os acionsitas, os clientes, os fornecedores e os stakeholders públicos (meio ambiente, comunidade, energia, caridade).

Com relação aos indicadores de CFP há autores que sugerem o emprego das métricas de CFP separadamente, como Lee et al. (2009), que empregaram testes separadamente para medidas contábeis e de mercado. Outros autores, a exemplo de Waddock \& Graves (1997), empregaram indicadores ROA, ROE, ROI de forma complementar para mensurar a performance financeira.

A relação entre CSP e CFP tem sido tema de investigações empíricas há pelo menos 20 anos. Conforme levantado por Boaventura et al. (2012), a dimensão CSP constitui-se em uma proxy que agrega os indicadores representativos dos diversos stakeholders: empregado, comunidade, cliente, fornecedor e investidor; a dimensão CFP constitui-se numa proxy que agrega diversos indicadores financeiros: ROA(Return on Asset), ROE (Return on Equity), ROI (Return on Investment), dividendos pagos, retorno acumulado, $\mathrm{Q}$ de Tobin.

Operacionalmente nesta pesquisa focaremos algumas variáveis mensuráveis que caracterizam CSP e CFP. Mais especificamente, para CSP, variáveis referentes aos stakeholders: empregados, clientes, meio ambiente, comunidade; e, para CFP, as variáveis: ROA, ROE e ROI.

\section{Metodologia de pesquisa}

\subsection{A metanálise}

A metanálise foi desenvolvida nos anos 70 por Hunter, Schmidt e Glass. Especificamente, Glass (1976) foi quem a divulgou inicialmente e a proposta de Hunter e Schmidt só apareceu publicada em 1982, a sua obra completa foi publicada em $1990 \mathrm{em}$ Hunter \& Schmidt (1990).

A metanálise é uma análise de análises de trabalhos empíricos - uma metodologia que acumula quantitativamente e integra resultados de pesquisas empíricas já realizadas (Gooding \& Wagner, 1985).

O objetivo geral do procedimento adotado para realizar a metanálise desenvolvida por Hunter \& Schmidt (1990) é agregar os coeficientes de correlação de uma amostra constituída de artigos com resultados empíricos que abordam uma determinada relação num campo de interesse do pesquisador. Hunter \& Schmidt (1990) argumentam que para realizar a metanálise é necessária uma amostra de dois ou mais artigos que abordam a relação de interesse. Inicialmente, o pesquisador deve estabelecer a relação entre variáveis que deseja analisar. Num segundo passo, o pesquisador deve estabelecer as condições de contorno da pesquisa - CODE (Gooding \& Wagner, 1985). Faz parte do CODE o nível da análise que configura a relação de interesse: se é em nível de pessoa, departamento ou organização; e a qualificação das variáveis de interesse. Em seguida, como terceiro passo, buscam-se os artigos que satisfazem o CODE estabelecido. Os artigos que atendem ao CODE estabelecido são reavaliados para confirmar a existência ou não do coeficiente de correlação entre as variáveis de interesse. Com a eliminação dos artigos que não contêm o coeficiente de correlação da relação de interesse se tem a coleção de artigos que formam a amostra da metanálise.

Assumindo que os coeficientes de correlação da relação de interesse sofrem a influência de artefatos criados pelo pesquisador que a realizou, para que eles possam ser aproveitados de forma acumulativa é necessário promover a eliminação de distorções associadas a esses artefatos (Hunter \& Schmidt, 1990). Hunter \& Schmidt (1990) distinguem os seguintes artefatos: (1) erro de amostragem que aumenta com a diminuição do tamanho da amostra do estudo; (2) erros resultantes de medição devidos ao uso de variáveis representativas não confiáveis do construto; (3) o espectro da amostra, que não reflete as características da população, inserindo viés; (4) erros de digitação e processamento dos dados; (5) contaminação e deficiência de medida; e (6) diferenças existentes entre alternativas de medição de um determinado construto.

Baseando-se num longo período de observação e análise de estudos realizados, Hunter \& Schmidt (1990) têm estimado que $72 \%$ da variância observada entre os estudos são oriundos do produto dos três primeiros artefatos. Dessa forma, Hunter \& Schmidt (1990) desenvolvem a metanálise a partir de cálculos que eliminam principalmente o erro de amostragem, pois os resultados obtidos nesse processo são suficientemente robustos para sustentarem conclusões que surgem pelo aproveitamento de pesquisas sobre uma relação de interesse de maneira cumulativa.

Assim, de posse da amostra com os artigos que contêm o coeficiente de correlação da relação de 
interesse, Hunter \& Schmidt (1990) propõem quatro etapas de cálculo e análise: (1) calcular a correlação média da amostra; (2) calcular a variância observada da correlação da amostra considerada e a variância devido ao erro de amostragem; (3) corrigir a variância da população pela subtração da variância devida ao erro de amostragem para estimação da correlação total; (4) analisar a existência dos efeitos moderadores, para que se criem subgrupos no sentido de tornar possível o discernimento de aspectos contextuais que estão influenciando a relação de interesse.

\subsubsection{Estimação da correlação média e a da variância}

A correlação média ponderada da amostra de artigos é obtida a partir da Equação 1.

$$
\bar{r}=\frac{\sum\left(N_{i}^{*} r_{i}\right)}{\sum N_{i}}
$$

Onde $\mathrm{N}$ é o número de elementos na amostra do estudo considerado e r é o coeficiente de correlação entre as variáveis de interesse obtidas no estudo.

Essa equação provê uma estimação acurada de medida para o coeficiente de correlação populacional, desde que estudos com amostras grandes são menos sujeitos ao erro de amostragem (Stevenson, 1981).

Para calcular a variância observada $\left(\sigma_{r}^{2}\right)$ entre os coeficientes de correlação individual através dos estudos, Hunter \& Schmidt (1990) propõem a utilização da média quadrática do erro ponderado pelo novo tamanho da amostra conforme a Equação 2.

$$
\sigma_{r}^{2}=\frac{\sum\left(N_{i} *\left(r_{i}-\bar{r}\right)^{2}\right)}{\sum N_{i}}
$$

Hunter \& Schmidt (1990) argumentam que a melhor estimativa da variância populacional não é a variância observada mas sim a variância observada menos a variância estimada, devido ao erro de amostragem. Consequentemente se faz necessário calcular a variância estimada devido ao erro de amostragem. conforme a Equação 3:

$$
\sigma_{e}^{2}=\frac{\left(1-\bar{r}^{2}\right)^{2} * k}{\sum N_{i}}
$$

$\mathrm{Na}$ Equação 3, k representa o número de estudos que foram considerados para o cálculo do coeficiente de correlação médio.

Assim, para a obtenção da variância da população basta subtrair o resultado obtido pela aplicação da Equação 3 do resultado obtido na Equação 2. A variância da população também é chamada de variância residual. Isso se deve ao fato de que, nos casos onde ela não é zero (ou aproximadamente zero), estará sinalizando a existência de efeitos moderadores que estão afetando a relação de interesse (Hunter \& Schmidt, 1990).

\subsection{A pesquisa para a metanálise}

As questões de pesquisa que orientaram as buscas de artigos, como colocadas anteriormente, são: "A predominância da relação positiva entre CSP e CFP ainda persiste em pesquisas mais recentes?" ou "As variáveis de quais stakeholders estão mais associadas à performance financeira?"

Como respostas a essas perguntas são formuladas as seguintes hipóteses:

- H1: É positiva a relação entre CSP e CFP nas pesquisas atuais.

- H2: Todos os stakeholders, representados por suas variáveis, se relacionam positivamente com a performance financeira.

Hunter \& Schmidt (1990) sugerem que seja estabelecida hipótese que admite a possibilidade de existência de variável moderada associada ao contexto de pesquisa. Por esse motivo, são estabelecidas as hipóteses H3.1 e H3.2, a saber:

\section{- H3.1: Existe variável moderadora afetando a relação CSP-CFP.}

- H3.2: Existe variável moderada afetando as relações entre as variáveis que representam cada stakeholder e a CFP.

Para desenvolver a metanálise adotou-se um intervalo de tempo que se inicia em 1998 e vai até 2010. Portanto, em sua essência está a utilização de trabalhos empíricos que tenham sido publicados nesse intervalo de tempo. Para levantamento dos artigos foi adotado o seguinte procedimento: escolha de palavras-chave, escolha das bases de dados, arquivamento dos artigos configurando uma amostra de artigos empíricos, seleção de artigos que apresentam o coeficiente de correlação entre as variáveis que compõem a relação CSP-CFP, incluindo as dimensões que compõem cada uma dessas proxies. Para identificar os artigos foram utilizadas as seguintes palavras-chave de busca: "financial performance", "corporate social performance". As bases de dados utilizadas foram: Proquest, Ebsco e ISI. Os artigos identificados por essas palavras-chave foram codificados (CODE) e formaram a amostra a ser analisada. Os artigos de interesse são aqueles que analisam a relação CSP-CFP em nível corporativo. Inicialmente foram identificados 58 artigos com resultados empíricos que apresentam tratamento estatístico dos dados, independentemente de usarem dados secundários ou primários. Para possibilitar a análise, os coeficientes 
de correlação foram computados de forma a permitir a identificação de a qual relação eles se referem: CSP-ROA, CSP-ROE, CSP-ROS, Empregado-ROA,
Cliente-ROA, Meio Ambiente-ROA, Comunidade-ROA. $\mathrm{O}$ resultado final foi uma amostra de 15 artigos que estão listados na Tabela 1.

Tabela 1. Amostra de artigos utilizados na metanálise de CSP-CFP.

\begin{tabular}{|c|c|c|c|c|c|c|}
\hline Autores/Ano & Periódico & $\mathbf{N i}$ & r observado & N. Rs & $\begin{array}{l}\text { Medidas de } \\
\text { CSP }\end{array}$ & $\begin{array}{l}\text { Medidas de } \\
\text { CFP }\end{array}$ \\
\hline $\begin{array}{l}\text { Bouslah et al. } \\
(2010)\end{array}$ & Journal of Business Ethics & 150 & 0,03 a 0,46 & 7 & $\begin{array}{l}\text { CSP, } \\
\text { Empregado, } \\
\text { Cliente, Meio } \\
\text { Ambiente, } \\
\text { Comunidade, } \\
\text { fornecedor e } \\
\text { investidor }\end{array}$ & $\begin{array}{c}\text { Retorno } \\
\text { sobre ativos } \\
\text { (ROA) }\end{array}$ \\
\hline Aras et al. (2010) & $\begin{array}{l}\text { International Journal } \\
\text { of Productivity and } \\
\text { Performance Management }\end{array}$ & 100 & 0,143 & 1 & Proxy CSP & ROA \\
\hline $\begin{array}{l}\text { Makni et al. } \\
(2009)\end{array}$ & Journal of Business Ethics & 179 & $-0,21$ a 0,18 & 6 & $\begin{array}{l}\text { CSP, } \\
\text { Empregado, } \\
\text { Meio } \\
\text { Ambiente, } \\
\text { Cliente }\end{array}$ & ROA e ROE \\
\hline $\begin{array}{l}\text { Elsayed \& Paton } \\
(2009)\end{array}$ & $\begin{array}{l}\text { Business Strategy \& the } \\
\text { Environment }\end{array}$ & 227 & 0,11 e 0,12 & 2 & $\begin{array}{l}\text { Meio } \\
\text { Ambiente }\end{array}$ & $\begin{array}{l}\text { Q de Tobin } \\
\text { e ROA }\end{array}$ \\
\hline $\begin{array}{l}\text { Choi \& Wang } \\
(2009)\end{array}$ & $\begin{array}{l}\text { Strategic Management } \\
\text { Journal }\end{array}$ & 1618 & 0,022 a 0,079 & 10 & $\begin{array}{l}\text { Comunidade, } \\
\text { Cliente, } \\
\text { Empregado, } \\
\text { Meio } \\
\text { Ambiente }\end{array}$ & $\begin{array}{l}\text { Q de Tobin } \\
\text { e ROA }\end{array}$ \\
\hline $\begin{array}{l}\text { Nelling \& Webb } \\
(2009)\end{array}$ & $\begin{array}{l}\text { Review of Quantitative } \\
\text { Finance \& Accounting }\end{array}$ & 600 & 0,066 & 1 & Proxy CSP & ROA \\
\hline $\begin{array}{l}\text { Mahoney et al. } \\
(2008)\end{array}$ & $\begin{array}{l}\text { Issues in Social } \\
\& \text { Environmental } \\
\text { Accounting }\end{array}$ & 44 & 0,$183 ; 0,189 ; 0,199$ & 3 & $\begin{array}{l}\text { Proxy CSP, } \\
\text { Cliente, } \\
\text { Empregado }\end{array}$ & ROA \\
\hline $\begin{array}{l}\text { Surroca \& Tribó } \\
(2008)\end{array}$ & $\begin{array}{l}\text { Journal of Business } \\
\text { Finance \& Accounting }\end{array}$ & 358 & $-0,09$ a 0,06 & 4 & $\begin{array}{l}\text { Proxy CSP, } \\
\text { Empregado }\end{array}$ & $\begin{array}{l}\text { Q de Tobin } \\
\text { e ROA }\end{array}$ \\
\hline $\begin{array}{l}\text { Van der Laan et al. } \\
(2008)\end{array}$ & Journal of Business Ethics & 2329 & $-0,14$ e 0,07 & 2 & Proxy CSP & ROA \\
\hline $\begin{array}{l}\text { Craig \& Dibrell } \\
(2006)\end{array}$ & Family Business Review & 217 & 0,1 & 1 & $\begin{array}{l}\text { Meio } \\
\text { Ambiente }\end{array}$ & ROA \\
\hline $\begin{array}{l}\text { Richard et al. } \\
(2006)\end{array}$ & $\begin{array}{l}\text { International Journal } \\
\text { of Human Resource } \\
\text { Management, }\end{array}$ & 574 & $-0,17$ e $-0,03$ & 2 & Comunidade & ROA e ROE \\
\hline Galbreath (2006) & Management Decision & 38 & $-0,47$ a 0,449 & 6 & $\begin{array}{l}\text { Empregado, } \\
\text { Meio } \\
\text { Ambiente, } \\
\text { Comunidade }\end{array}$ & ROA e ROE \\
\hline Ruf et al. (2001) & Journal of Business Ethics & 400 & 0,089 & 1 & Proxy CSP & ROE \\
\hline $\begin{array}{l}\text { Berman et al. } \\
(1999)\end{array}$ & $\begin{array}{l}\text { Academy of Management } \\
\text { Journal }\end{array}$ & 81 & 0,$01 ; 0,04 ; 0,10 ; 0,22$ & 4 & $\begin{array}{l}\text { Empregado, } \\
\text { Cliente, Meio } \\
\text { Ambiente, } \\
\text { Comunidade }\end{array}$ & ROA \\
\hline $\begin{array}{l}\text { Graves \& } \\
\text { Waddock (1999) }\end{array}$ & $\begin{array}{l}\text { International Journal of } \\
\text { Value-Based Management }\end{array}$ & 500 & 0,08 a 0,98 & 5 & $\begin{array}{l}\text { Proxy CSP, } \\
\text { Empregado, } \\
\text { Cliente, Meio } \\
\text { Ambiente, } \\
\text { Comunidade }\end{array}$ & ROA \\
\hline
\end{tabular}


Pela Tabela 1 pode ser constatado que apesar do intervalo de pesquisa ser de 1998 a 2010, a amostra contém artigos a partir de 1999 até 2010. Isso se deve ao fato de os artigos de datas anteriores não conterem coeficientes de correlação de algum par CSP-CFP de interesse. Na Tabela 1 há também a indicação de três artigos que fornecem o coeficiente de correlação entre CSP e q de Tobin, entretanto os três coeficientes de correlação fornecidos medem associações com diferentes indicadores da CSP, não sendo possível realizar a metanálise para a relação CSP-q de Tobin.

\subsubsection{Resultados obtidos}

Aplicando o método de tratamento de dados proposto por Hunter \& Schmidt (1990), obtém-se o resultado apresentado na Tabela 2.

$\mathrm{O}$ valor de $\mathrm{k}$ representa a quantidade de estudos considerados para a determinação do valor agregado de $r$ e de suas respectivas variâncias; o tamanho da amostra é resultado da soma das amostras de cada estudo empírico; r é o coeficiente de correlação médio ponderado obtido a partir dos coeficientes de correlação de cada estudo considerado. A variância observada é a variância calculada tendo como referência o coeficiente de correlação médio e a respectiva correlação de cada um dos estudos considerados. A variância residual é a variância da população, que por conceito deve ser zero, e a variância explicada é a variância oriunda do erro de amostragem (artefato 1).
Os resultados apresentados nas Tabelas 2 e 3 foram submetidos à avaliação de viés proposta por Rosenthal (1979). Rosenthal (1979) sugere que a existência de viés no resultado total dos estudos sumarizados seja investigada a partir do nível de significância estatística de cada elemento da amostra. Utilizando sua proposta para analisar todas as relações, para a relação CSP-ROS foi indicada a necessidade de totalizar 75 estudos para alterar substancialmente o resultado apresentado para essa relação na Tabela 1. Para as outras relações, o número de estudos foi superior a 75 , confirmando a robustez dos resultados obtidos.

A partir dos cálculos realizados sobre os dados dos artigos que compõem a amostra, pode-se constatar que as relações: CSP-ROA, CSP-ROE, Cliente-ROA, Meio Ambiente-ROA, Comunidade-ROA apresentam relação neutra, ou seja, não se confirma a relação positiva entre elas. As respectivas variâncias observadas para essas relações estão próximas de zero e isso reafirma a neutralidade dessas relações, ainda que possa haver uma explicação devido ao erro de amostragem. Como é o caso da relação CSP-ROE, que apresenta uma variância devida ao erro de amostragem, que explica a variabilidade em $51,02 \%$ do r $(0,0784)$ associado, e da relação Ambiente-ROA, que apresenta uma variância devida ao erro de amostragem, que explica em $71,66 \%$ a variabilidade do r $(0,07699)$ associado. Portanto, ainda que se façam novas pesquisas abordando essas relações, é de se esperar como resultado a neutralidade, ou seja, as dimensões que compõem essas relações não são correlacionadas

Tabela 2. Resultado obtido da metanálise com todas as relações de interesse.

\begin{tabular}{cccccccc}
\hline Relação & $\mathbf{k}$ & $\begin{array}{c}\text { Tamanho total } \\
\text { da amostra }\end{array}$ & $\mathbf{r}^{1}$ & $\begin{array}{c}\text { Variância } \\
\text { observada }^{2}\end{array}$ & $\begin{array}{c}\text { Variância } \\
\text { populacional }^{3}\end{array}$ & $\begin{array}{c}\text { \% } \\
\text { variância } \\
\text { explicada }\end{array}$ & $\begin{array}{c}\text { Sig. para } \\
\mathbf{r}^{*}\end{array}$ \\
\hline CSP-ROA & 8 & 6589 & 0,00114 & 0,016 & 0,01465 & 8,44 & 0,000967 \\
CSP-ROE & 3 & 679 & 0,0784 & 0,00855 & 0,004188 & 51,02 & 0,0156 \\
CSP-ROS & 3 & 1000 & 0,1026 & 0,00007 & $-0,00286$ & 0,00 & 0,0267 \\
Empregado-ROA & 8 & 2968 & 0,2126 & 0,1221 & 0,1196 & 2,05 & 0,000893 \\
Cliente-ROA & 6 & 2572 & 0,0679 & 0,00574 & 0,0034 & 40,77 & 0,00987 \\
Meio Ambiente-ROA & 8 & 3010 & 0,07699 & 0,00367 & 0,00104 & 71,66 & 0,00897 \\
Comunidade-ROA & 6 & 2566 & 0,0724 & 0,0082 & 0,00585 & 28,66 & 0,00998 \\
\hline
\end{tabular}

${ }^{1}$ Calculado utilizando-se a Equação $1 ;{ }^{2} \mathrm{Calculado}$ utilizando-se a Equação $2 ;{ }^{3} \mathrm{Calculado}$ realizando-se a diferença de ${ }^{2} \mathrm{com}^{3} ; *$ Foi utilizada a sistemática de cálculo proposta por Rosenthal (1979). Fonte: autores.

Tabela 3. Resultado da metanálise para os subgrupos de Empregado-ROA.

\begin{tabular}{cccccccc}
\hline Relação & k & $\begin{array}{c}\text { Tamanho total } \\
\text { da amostra }\end{array}$ & $\mathbf{r}$ & $\begin{array}{c}\text { Variância } \\
\text { observada }\end{array}$ & $\begin{array}{c}\text { Variância } \\
\text { populacional }\end{array}$ & $\begin{array}{c}\text { \% } \\
\text { variância } \\
\text { explicada }\end{array}$ & $\begin{array}{c}\text { Sig. para } \\
\mathbf{r}\end{array}$ \\
\hline Empregado-ROA 1 & 3 & 3665 & 0,054618 & 0,001984 & 0,00117 & 41,01 & 0,00896 \\
Empregado-ROA 2 & 4 & 718 & 0,233125 & 0,001061 & $-0,00392$ & 0,00 & 0,00899 \\
\hline
\end{tabular}

Fonte: autores. 
diretamente, para níveis de significância iguais a $0,05<\mathrm{p}$ (ou menores).

A relação CSP-ROS apresenta uma pequena correlação $(0,102)$ e é um resultado que em princípio não está afetado por variáveis moderadoras, pois a variância populacional é zero, com nível de significância de $0,05<\mathrm{p}$.

A relação Empregado-ROA apresenta um coeficiente de correlação de 0,2126 , pequeno mas com algum significado. Além disso, a variância populacional não é diferente de zero $(0,1196)$, apontando para a existência de variáveis moderadoras que intervêm nesse coeficiente. Dessa forma, é necessário subdividir a amostra que compõe essa relação.

O conjunto de oito artigos que representam a relação Empregado-ROA foi dividido em dois subgrupos: ROA 1, com três artigos, e ROA 2, com quatro artigos e um artigo foi desconsiderado. Com isso, foi possível gerar a análise que está apresentada na Tabela 3.

Observe na Tabela 3 que um subgrupo é composto por correlações pequenas, ROA 1, e outras por correlações significativas, ROA 2.

Os dois subgrupos configurados não contêm o artigo de Graves \& Waddock (1999), que apresentou um coeficiente de correlação entre Empregado-ROA de 0,98 . De fato, esse artigo propositalmente explora essa relação por um viés, ou seja, fizeram parte da amostra estudada empresas da lista publicada pela revista Fortune denominada America's Most Admired Corporations. Além disso, para a dimensão financeira foi utilizado o retorno financeiro total (médio) de 10 anos, também proposto pela revista Fortune, diferentemente dos outros artigos da amostra para essa relação em questão. Assim, é de se esperar que a relação Empregado-ROA expresse alta correlação, confirmando o resultado.

Com a subdivisão do grupo inicial que compõe a relação Empregado-ROA foi possível perceber um grupo em que a relação é neutra $(r=0,054618)$ e um grupo com valor de $r$ igual a 0,233125 . Para o primeiro grupo, a variância é em $41,01 \%$ explicada pelo erro de amostragem, para o segundo grupo, a variância populacional é zero, não havendo variância devido ao erro de amostragem.

Os três artigos que subsidiaram o cálculo de $\mathrm{r}$ para o subgrupo 1 (Empregado-ROA 1) possuem peculiaridades contextuais que merecem destaque $\mathrm{e}$ que apontam diferentes variáveis moderadoras que interferiram no resultado obtido para o coeficiente de correlação. Makni et al. (2009) obtiveram um coeficiente de correlação para essa relação de 0,09 , em sua amostra estão empresas de pequeno porte do Canadá. Além disso, os autores argumentam que essas empresas precisam de apoio governamental para promover investimentos em dimensões do CSP. Choi \& Wang (2009), para compor a amostra de seu estudo, configuram dois grupos de interesse, um com ROA acima da média do setor e outro com ROA abaixo da média do setor. Esses dois subgrupos foram consolidados para determinar os coeficientes de correlação, consequentemente, o valor de $r$ tendeu a zero. Por fim, Surroca \& Tribó (2008) vão medir a dimensão "empregado", por uma proxy que representa a sua satisfação, a qual é composta por 37 indicadores que cobrem diferentes aspectos da empresa que envolve os temas vinculados ao empregado. Uma medida que representa a percepção do empregado em relação ao sistema de gestão como um todo.

$\mathrm{O}$ que se pode constatar disso é que diferentes variáveis moderadoras interferiram na relação Empregado-ROA. Portanto, o subgrupo 1 deve ser tratado com distinção e não deve ser adicionado ao subgrupo 2 (Empregado-ROA 2).

No subgrupo 2 não foram encontrados aspectos que pudessem sugerir a subdivisão ou anulação do resultado obtido. Além disso, entre os artigos que compõem o segundo grupo (Empregado-ROA2), os que obtiveram os maiores coeficientes de correlação foram aqueles que fizeram a análise considerando a dimensão social (Empregado) atrasada (lag) de um ano em relação à dimensão financeira (ROA). Os quatro artigos desse subgrupo são estudos realizados em países diferentes, focando empresas de Índia, Austrália e Estados Unidos. Em particular, o artigo de Mishra \& Suar (2010), que foca empresas indianas, utilizou um questionário elaborado por um processo sistemático. Baseou-se na ferramenta de coleta utilizada pelo KLD (Kinder, Lydenberg, Domini), considerada uma das ferramentas mais confiáveis de coleta de informação sobre responsabilidade social (Hillman \& Keim, 2001). Após a elaboração da sua primeira versão, ela foi submetida a especialistas da área para críticas e sugestões. O questionário revisado passou por pré-teste e ainda sofreu ajustes, para ser então distribuído. Dessa forma, apenas um dos artigos da amostra utilizou um instrumento de coleta de informação diferente do instrumento utilizado pela KLD, que é o artigo de Galbreath (2006). Galbreath (2006) fez uso do índice denominado RepuTex. O instrumento de coleta de dados que alimenta o RepuTex tem como seus idealizadores especialistas acadêmicos da área, de organizações não governamentais e de empresas sem fins lucrativos (Galbreath, 2006). Portanto, não são encontradas evidências objetivas da existência de variáveis moderadoras, consequentemente, confirma-se a existência de relação entre a variável Empregado e o ROA. Ou seja, atender aos interesses dos Empregados correlaciona-se com a performance financeira ROA.

Dessa forma, a hipótese 1 foi parcialmente refutada, na medida em que as respectivas relações entre as dimensões de CSP-CFP de maneira geral não apresentaram relação positiva, mas neutra. Apenas a relação CSP-ROS apresenta $r=0,102$, com nível 
de significância estatística de $0,05<$ p. Portanto, não se pode afirmar de maneira contundente que a relação CSP-CFP se mantém positiva nos estudos atuais. $\mathrm{O}$ mesmo acontece para a $\mathrm{H} 2$, ou seja, não se pode afirmar de maneira contunde que a relação é positiva para a relação entre todos os stakeholders e a CFP. Apenas a relação Empregado-ROA apresenta relação positiva.

Para as hipóteses que se referem à existência de variável moderada, a hipótese H3.1 não se confirmou, ou seja, não foi constatada existência de variável moderadora na relação CSP-CFP, mas a hipótese H3.2 foi parcialmente confirmada, na medida em que foi contatada a existência de moderação na relação Empregado-ROA.

\subsubsection{As implicações práticas e teóricas}

Os resultados obtidos pela metanálise geram diferentes implicações, a saber: as condições de pesquisa que dão robustez aos dados coletados e, a partir desses resultados, os aspectos teóricos relacionados à relação CSP-CFP.

A metanálise realizada possibilitou reforçar aspectos sobre as condições para realização da coleta de dados, no sentido de se respeitar não só as premissas estatísticas de aleatoriedade dos elementos da amostra e a representatividade da população de interesse, pela amostra obtida, mas também a utilização de instrumentos de coleta de dados que sejam construídos respeitando os construtos conceituais de interesse. Algumas relações analisadas apresentam variâncias elevadas devido a artefatos de pesquisa. É desejável que esforços sejam orientados para a diminuição de interferências dessa natureza.

Em termos práticos, para a gestão de stakeholders, a metanálise também evidenciou diferenças na relação entre CSP-CFP. Entre as relações avaliadas com a proxy CSP, a única relação que apresentou relação positiva foi a CSP-ROS, as outras, CSP-ROA, CSP-ROE, apresentaram relação neutra. Todas com nível de significância estatística aceitável. Portanto, a orientação de que as empresas devam investir em CSP para melhorarem a sua CFP deve ser visto com parcimônia, pois o resultado final pode não se confirmar, pois vai depender muito do tipo de resultado financeiro que está sendo pretendido.

Em termos das dimensões (Comunidade, Empregado, Cliente, Meio Ambiente e as medidas de performance financeira), essas diferenças colocam em destaque a relação Empregado-ROA, que apresenta correlação positiva, enquanto as outras relações analisadas caracterizam relações de neutralidade. Essa relação é ainda mais destacada das outras quando se analisa empresas que constam de relações de empresas tipo The Most Admired Corporations. Assim, a orientação prática de que investir em funcionários promove resultados financeiros pode se configurar como uma orientação assertiva em termos da dimensão Empregado, configurada entre as dimensões componentes do CSP.

Em termos de teoria instrumental de stakeholder, as relações de neutralidades entre CSP e CFP reforçam a necessidade de se estabelecer outra lógica conceitual de abordagem, que possa ser utilizada em pesquisa empírica. Dessa forma, impõe-se a reconstrução da relação causal CSP-CFP. Uma proposta de raciocínio é desenvolver relações não lineares entre essas duas proxies. O mesmo podendo ser explorado para as outras relações: Comunidade-ROA, Cliente-ROA, Meio Ambiente-ROA, entre outras. O fato de não existir correlação entre CSP e CFP não significa que elas não sejam relacionadas, apenas indica que a relação linear não se faz presente, podendo, inclusive, existirem variáveis intervenientes que interferem na relação.

\section{Conclusão}

A questão geral de pesquisa sobre ser ou não ser positiva a relação entre CSP e CFP foi avaliada utilizando-se a metanálise. Os resultados apontam em termos gerais e específicos para uma relação neutra entre CSP e CFP, com poucas exceções. Ou seja, os resultados não generalizam uma relação positiva entre CSP e CFP. Em termos específicos, CSP-ROS se mostrou (levemente) correlacionada positivamente. Continuam neutras as relações Cliente-ROA, Comunidade-ROA, Meio Ambiente-ROA.

Um ponto relevante a ser observado é que a relação Empregado-ROA apresenta-se como positiva para um subgrupo de artigos que, em termos metodológicos, foram mais rigorosos para coletar dados que mensurassem essa relação. Esse resultado deve ser ressaltado, uma vez que mesmo aplicado um rigor na análise dessa relação a mesma persiste, ou seja, os resultados reforçam que uma associação entre o desempenho organizacional junto aos funcionários e o respectivo desempenho financeiro da empresa. Para um segundo subgrupo, essa relação se manteve neutra por motivos que justificam o resultado. Ora porque a pesquisa teve sua amostra composta por empresas pequenas, com poucos recursos para investir nas dimensões do CSP, ora porque o estudo quis comparar empresas de alto desempenho com empresas de baixo desempenho. E quando o estudo foca empresas tipo The Most Admired Corporations, a relação Empregado-ROA apresenta um alto coeficiente de correlação, dando a entender que é possível se pensar na possibilidade de existência de causalidade entre as variáveis que definem a relação.

São poucos os estudos que empregam metanálise estatística na averiguação da relação CSP/CFP e, principalmente, apresentando de forma didática e replicável esse método. Nesse sentido, este trabalho contribui para a literatura revelando passo a passo 
como tal técnica pode ser empregada para esse fim e dessa forma tornando-se útil para pesquisadores com interesse em futuros estudos congêneres.

As implicações desses resultados contribuem para o avanço da teoria dos stakeholders ao apontar para a existência de relação não linear entre CSP e CFP, pelo menos em termos de variáveis específicas que compõem essas duas proxies.

Nesse sentido, é necessário investigar os microfundamentos da relação CSP-CFP, especificamente os da teoria instrumental de stakeholders. É necessário repensar a relação linear entre essas proxies. Considerar relações não lineares gera novas perspectivas para a teoria instrumental de stakeholder. Isso retira do tratamento da relação CSP-CFP o caráter simplista adotado até o momento.

A relação CSP-ROS se mostrou levemente correlacionada, e isso deve nos inspirar para identificar de fato que variáveis de CSP correlacionam-se com o ROS, tendo em vista que a proxy CSP é resultante de uma equação matemática que pondera as suas próprias variáveis. Assim, é preponderante que novas pesquisas explorem a natureza de cada variável escolhida para representar cada um dos stakeholders na equação que resulta o cálculo da CSP.

A relação Empregado-ROA que teve correlação positiva já indica que para efeitos de formulação e implementação da estratégia de produção, a perspectiva bottom-up tem relevância intrínseca, na medida em que essa perspectiva pode incorporar demandas apresentadas pelos colaboradores do nível operacional, e como decorrência, se elas forem atendidas, é provável que isso se reflita numa melhor performance de ROA. Novas investigações dessa relação podem confirmar se há relação de causalidade entre Empregados e ROA e em que condições isso ocorre, reforçando a necessidade de se reconhecer o ciclo virtuoso: atendimento dos interesses de colaboradores-performance operacional-atendimento dos interesses de clientes-desempenho financeiro-atendimento dos interesses de colaboradores.

\section{Referências}

Aras, G., Aybars, A., \& Kutlu, O. (2010). Managing corporate performance: investigating the relationship between corporate social responsibility and financial performance in emerging markets. International Journal of Productivity and Performance Management, 59(3), 229-254. http://dx.doi.org/10.1108/17410401011023573.

Aupperle, K. E., Carrol, A. B., \& Hatfield, J. D. (1985). An empirical examination of the relationship between corporate social responsibility and profitability. Academy of Management Journal, 28(2), 446-463.

Barnett, M. L. (2007). Stakeholder influence capacity and the variability of financial returns to corporate social respons. Academy of Management Review, 32(3), 794816. http://dx.doi.org/10.5465/amr.2007.25275520.

Berman, S. L., Wicks, A. C., Kotha, S., \& Jones, T. M. (1999). Look at the financial-social performance nexus when quality of management is held constant. Academy of Management Journal, 42(5), 488-506.

Bird, R., Hall, D. A., Momentè, F., \& Reggiani, F. (2007). What corporate social activities are valued by the market? Journal of Business Ethics, 76(2), 189-206. http://dx.doi.org/10.1007/s10551-006-9268-1.

Boaventura, J. M. G., Silva, R. S., \& Bandeira-de-Mello, R. (2012). Performance financeira corporativa e performance social corporativa: desenvolvimento metodológico e contribuição teórica dos estudos empíricos. Revista Contabilidade \& Finanças, 23(60), 232-245. http:// dx.doi.org/10.1590/S1519-70772012000300008.

Bouslah, K., Zali, B., Turcotte, M. F., \& Kooli, M. (2010). The impact of forest certification on firm financial performance in Canada and the U.S. Journal of Business Ethics, 96(4), 551-572.

Callan, S. J., \& Thomas, J. M. (2009). Corporate financial performance and corporate social performance. Corporate Social Responsibility and Environmental Management, 16(2), 61-78. http://dx.doi.org/10.1002/csr.182.

Carroll, A. (1979). A three-dimensional model of corporate performance. Academy of Management Review, 4(4), 497-505. http://dx.doi.org/10.5465/amr.1979.4498296.

Chih, H.-L., Chih, H.-H., \& Chen, T.-Y. (2010). On the determinants of corporate social responsibility: international evidence on the financial industry. Journal of Business Ethics, 93(1), 115-135. http://dx.doi.org/10.1007/ s10551-009-0186-X.

Choi, J., \& Wang, H. (2009). Stakeholder relations and the persistence of corporate financial performance. Strategic Management Journal, 30(4), 895-907. http:// dx.doi.org/10.1002/smj.759.

Clarkson, M. B. E. (1995). A stakeholder framework for analyzing and evaluating corporate social performance. Academy of Management Review, 20(1), 92-117. http:// dx.doi.org/10.5465/amr.1995.9503271994.

Coase, R. H. (1937). The nature of the firm. Economica, 4(16), 386-405. http://dx.doi.org/10.1111/j.1468-0335.1937. tb00002.x.

Cochran, P. L., \& Wood, R. A. (1984). Corporate social responsibility and financial performance. Academy of Management Journal, 27(1), 42-56.

Coombs, J. E., \& Gilley, K. M. (2005). Stakeholder management as predictor of CEO compensation: main effects and interaction with financial performance. Strategic Management Journal, 26(9), 827-840. http:// dx.doi.org/10.1002/smj.476.

Craig, J., \& Dibrell, C. (2006). The natural environment, innovation, and firm performance: a comparative study. 
Family Business Review, 19(4), 275-288. http://dx.doi. org/10.1111/j.1741-6248.2006.00075.x.

Elsayed, K., \& Paton, D. (2009). The impact of financial performance on environmental policy: does firm life cycle matter? Business Strategy and the Environment, 18(6), 397-413. http://dx.doi.org/10.1002/bse.608.

Evan, W. M., \& Freeman, R. E. (1993). A stakeholder theory of the modern corporation: Katian capitalism. In T. Donaldson \& P. H. Werhane (Eds.), Ethical issues in business (pp. 166-171). Englewood Cliffs: Prentice-Hall.

Fauzi, H., \& Idris, K. M. (2009). The relationship of CSR and financial performance: new evidence from Indonesian companies. Issues in Social and Environmental Accounting, 3(1), 66-87. http://dx.doi.org/10.22164/isea.v3i1.38.

Freeman, R. E. (1984). Strategic management: a stakeholder approach. Miane: Pitman.

Friedman, M. (1970). The social responsibility of business is to increase its profits. New York Times Magazine, 33-35.

Frooman, J. (1999). Stakeholder influences strategies. Academy of Management Review, 24(2), 191-205. http:// dx.doi.org/10.5465/amr.1999.1893928.

Galbreath, J. (2006). Does primary stakeholder management positively affect the bottom line? Some evidence from Australia. Management Decision, 44(8), 1106-1121. http://dx.doi.org/10.1108/00251740610690649.

Glass, G. V. (1976). Primary, secondary and meta-analysis of research. Educational Researcher, 5(10), 3-8. http:// dx.doi.org/10.3102/0013189X005010003.

Gooding, R. Z., \& Wagner, J. A., 3rd. (1985). A metaanalytic review of the relationship between size and performance: The productivity efficiency of organizations and their subunits. Administrative Science Quarterly, 30(4), 462-481. http://dx.doi.org/10.2307/2392692.

Graves, S. B., \& Waddock, S. A. (1999). A look at the financial-social performance nexus when quality of management is held constant. International Journal of Value-Based Management, 12(1), 87-99. http://dx.doi. org/10.1023/A:1007770406555.

Hayes, R. H., \& Wheelwright, S. C. (1984). Restoring our competitive edge. Nova Iorque: John Wiley.

Hillman, P., \& Keim, G. D. (2001). Stakeholder value, stakeholder management, and social issues: what's the bottom line? Strategic Management Journal, 22(2), 125-139. http://dx.doi.org/10.1002/10970266(200101)22:2<125::AID-SMJ150>3.0.CO;2-H.

Hopkins, M. (1997). Defining indicators to assess socially responsible enterprises. Futures, 29(7), 581-603. http:// dx.doi.org/10.1016/S0016-3287(97)00034-7.

Hunter, J. E., \& Schmidt, F. L. (1990). Methods of metaanalysis: Correcting and bias in research findings. Londres: Sage.

Jensen, M. (2001). Value maximization, stakeholder theory, and the corporate objective function. Journal of
Applied Corporate Finance, 14(3), 8-21. http://dx.doi. org/10.1111/j.1745-6622.2001.tb00434.x.

Jones, T. M. (1995). Instrumental stakeholder theory: a synthesis of ethics and economics. Academy of Management Review, 20(2), 404-437. http://dx.doi. org/10.5465/amr.1995.9507312924.

Lee, D. D., Faff, R. W., \& Langfield-Smith, K. (2009). Revisiting the vexing question: does superior corporate social performance lead to improved financial performance? Australian Journal of Management, 34(1), 21-49. http:// dx.doi.org/10.1177/031289620903400103.

Lougee, B., \& Wallace, J. (2008). The corporate social responsibility (CSR) trend. Journal of Applied Corporate Finance, 20(1), 96-108. http://dx.doi.org/10.1111/j.17456622.2008.00172.x.

Mahoney, L., LaGore, W., \& Scazzero, J. A. (2008). Corporate social performance, financial performance for firms that restate earnings. Issues in Social and Environmental Accounting, 2(1), 104-130. http://dx.doi. org/10.22164/isea.v2i1.27.

Makni, R., Francoeur, C., \& Bellavance, F. (2009). Causality between corporate social performance and financial performance: Evidence from Canadian firms. Journal of Business Ethics, 89(3), 409-422. http://dx.doi. org/10.1007/s10551-008-0007-7.

Margolis, J. D., \& Walsh, J. P. (2003). Misery loves companies: rethinking social initiatives by business. Administrative Science Quarterly, 48(2), 268-305. http://dx.doi.org/10.2307/3556659.

Mazis, M., \& Green, R. (1971). Implementing social responsibility. MSU Business Topics, 68-76.

Mishra, S., \& Suar, D. (2010). Does corporate social responsibility influence firm performance of Indian companies? Journal of Business Ethics, 95(4), 571-601. http://dx.doi.org/10.1007/s10551-010-0441-1.

Nelling, E., \& Webb, E. (2009). Corporate social responsibility and financial performance: the "virtuous circle" revisited. Review of Quantitative Finance and Accounting, 32(2), 197-209. http://dx.doi.org/10.1007/s11156-008-0090-y.

Orlitzky, M., Schmidt, F. L., \& Rynes, S. L. (2003). Corporate social and financial performance: a metaanalysis. Organization Studies, 24(3), 403-441. http:// dx.doi.org/10.1177/0170840603024003910.

Porter, M. E. (1980). Competitive strategy. New York: Free Press.

Richard, O. C., Ford, D., \& Ismail, K. (2006). Exploring the performance effects of visible attribute diversity: the moderating role of span of control and organizational life cycle. International Journal of Human Resource Management, 17(12), 2091-2109. http://dx.doi. org/10.1080/09585190601000246.

Rosenthal, R. (1979). The File Drawer Problem and tolerance for nulls results. Psychological Bulletin, 86(3), 638-641. http://dx.doi.org/10.1037/0033-2909.86.3.638. 
Ruf, B. M., Muralidhar, K., Brown, R. M., Janney, J. J., \& Paul, K. (2001). An Empirical Investigation of the Relationship Between Change in Corporate Social Performance and Financial Performance: A Stakeholder Theory Perspective. Journal of Business Ethics, 32(2), 143-156. http://dx.doi.org/10.1023/A:1010786912118.

Slack, N., Chambers, S., \& Johnston, R. (2002). Administração da produção. São Paulo: Atlas.

Stevenson, W. J. (1981). Estatística aplicada à administração. São Paulo: Harbra.

Surroca, J., \& Tribó, J. A. (2008). Managerial entrenchment and corporate social performance. Journal of Business Finance \& Accounting, 35(5), 748-789. http://dx.doi. org/10.1111/j.1468-5957.2008.02090.x.

Ullmann, A. A. (1985). Data in search of a theory: a critical examination of economic performance on U.S. firms. Academy of Management Review, 10(3), 540-557.

Van Beurden, P., \& Gössling, T. (2008). The worth of values: a literature review on the relation between corporate social and financial performance. Journal of Business Ethics, 82(2), 407-424. http://dx.doi.org/10.1007/ s10551-008-9894-X.

Van der Laan, G., Van Ees, H., \& Van Witteloostuijn, A. (2008). Corporate social and financial performance: an extended stakeholder theory, and empirical test with accounting measures. Journal of Business Ethics, 79(3), 299-310. http://dx.doi.org/10.1007/s10551-007-9398-0.

Waddock, S. A., \& Graves, S. B. (1997). The corporate social performance-financial performance link. Strategic Management Journal, 18(4), 303-319. http://dx.doi. org/10.1002/(SICI)1097-0266(199704)18:4<303::AIDSMJ869>3.0.CO;2-G.

Whetten, D. A., Rands, G., \& Godfrey, P. (2002). What are the responsibilities to society? In: Pettigrew, A., Thomas, H., \& Whittington, R. Handbook of strategy and management. Londres: Sage.

Wood, D. J. \& Jones, R. (1995). Stakeholder mismatching: a theoretical problem in empirical research on corporate social performance. The International Journal of Organizational Analysis, 3(3), 229-267.

Wood, D. J. (1991). Corporate social performance revisited. Academy of Management Review, 16(4), 691-718. http:// dx.doi.org/10.5465/amr.1991.4279616.

Wood, D. J. (2010). Measuring corporate social performance: a review. International Journal of Management Reviews, 1(1), 50-84. http://dx.doi.org/10.1111/j.14682370.2009.00274.x. 